\title{
Percutaneous Endovascular Recanalization of a Thrombosed Aortic Graft Limb with Stent Placement
}

\author{
Efstratios Georgakarakos, MD*, Konstantinos Kapoulas, MD \\ Department of Vascular Surgery, "Democritus” University of Thrace, University Hospital of Alexandroupolis, Alexandroupolis, Greece
}

\begin{abstract}
Common practice in recanalization of a thrombosed prosthetic graft limb in an aortoiliac bypass focuses on balloon-catheter thrombectomy and angiographic exploration followed either by open surgical revision or endovascular management. This report describes the technique of percutaneous endovascular recanalization of an early thrombosed aortic graft limb with stent placement and subsequent restoration of patency and adequate limb perfusion, which remains patent after one year. Percutaneous intervention with stent placement and angioplasty for early graft limb recanalization avoids femoral incisions, and complications or morbidity associated with open surgery, while permitting rapid mobilization of the patient.

Copyright $\odot 2014$ Science International Corp.
\end{abstract}

\section{Key Words}

Aorta - Endovascular - Iliac artery - Graft . Atherosclerosis

\section{Introduction}

Prosthetic limb occlusion of an aortic bifurcated graft can be attributable to a variety of causes, leading to lower limb ischemia. The underlying reasons for immediate occlusion include technical faults or underlying thrombophilia (within the first postoperative month). Later events are attributed to intimal hyperplasia at the level of the peripheral anastomosis (oc-

\author{
(C) 2014 Aorta. \\ Published by Science International Corp. \\ ISSN 2325-4637
}

Fax +1 2037853346

E-Mail: aorta@scienceinternational.org

http://aorta.scienceinternational.org curring from $2 \mathrm{mo}$ to $3 \mathrm{yr}$ postoperatively) or to progression of the atherosclerotic disease, thrombosis of a false aneurysm at the anastomosis, graft degeneration, elongation, and kinking [1].

The most common therapeutic practice for a failed aortofemoral bypass focuses on surgical incision, arteriotomy, and open thrombus removal with balloonthrombectomy followed by angiographic exploration of the underlying cause and endovascular or surgical repair [1]. Endovascular management is the preferred approach for failed aortobiliac grafts, provided that the distal anastomosis can be crossed with a wire. Thrombolytic or pharmacomechanical modalities have also been described [2].

In this report, we describe a case of percutaneous endovascular recanalization of a thrombosed aortoiliac graft limb with angioplasty and stent placement with subsequent restoration of patency and adequate limb perfusion.

\section{Case Presentation}

A 55-year-old male patient was subjected to aortobiliac bypass with a bifurcated Dacron graft, $20 \times$ $10 \mathrm{~mm}$, due to occlusion of the infrarenal aorta with patent iliac vessels. Femoral pulses were immediately restored. Given a negative past history of atrial fibrillation, arrhythmia, or thrombophilia, the patient was put on antiplatelet monotherapy. He was shortly dis-

*Corresponding author:

Efstratios Georgakarakos, MD

Department of Vascular Surgery

University Hospital of Alexandroupolis

68100, Alexandroupolis, Greece

Tel: +1 0030 6945891236, Fax: +1 003025510 76124, E-Mail: efstratiosgeorg@gmail.com 

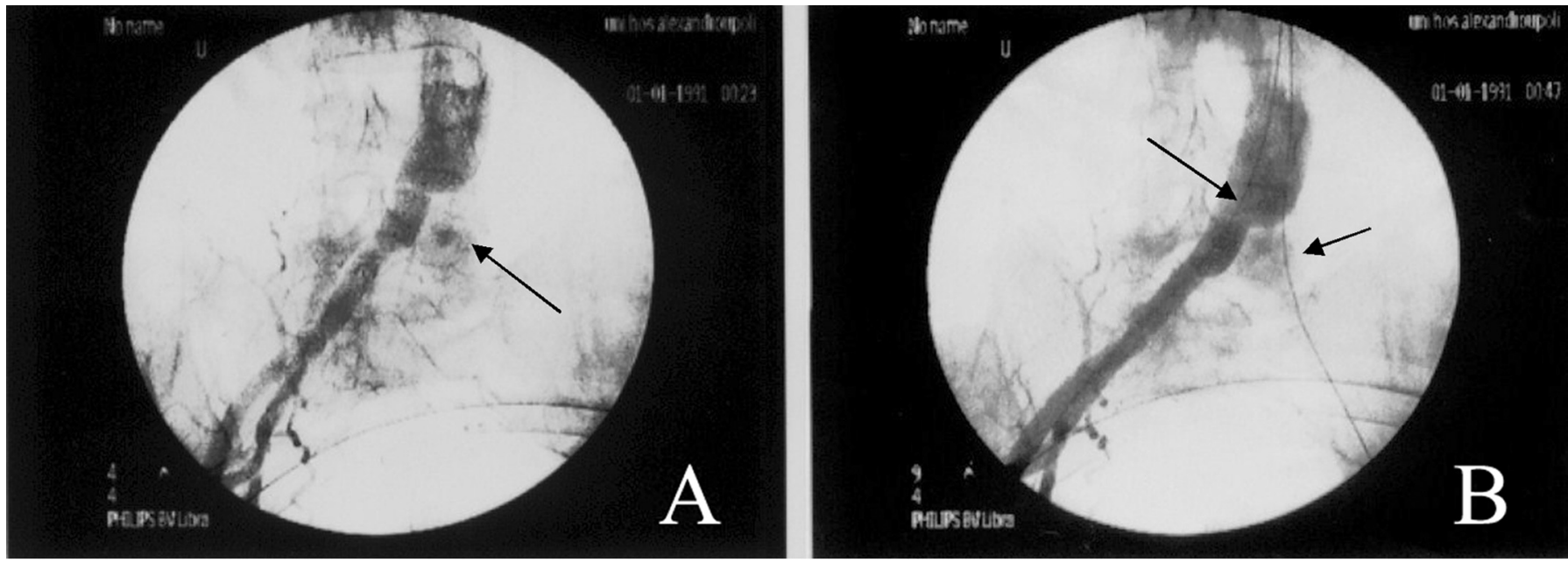

Figure 1. (A) Flush occlusion (arrow) of the left limb of an aortoiliac bifurcated bypass graft. (B) Hydrophilic guide wires (arrows) positioned within both graft limbs.

charged with fine, palpable femoral pulses bilaterally and returned to his ordinary activity. He returned 3 mo later, complaining of recurrence of intermittent claudication of the left lower limb, presenting no palpable pulses over the ipsilateral common femoral artery. The medical history revealed that his first symptoms appeared suddenly, shortly after his discharge, but were falsely attributed to musculoskeletal causes, resulting in delayed admission.

We proceeded directly with intraoperative angiography, in order to therapeutically intervene immediately after detection of the underlying cause. A 7Fx $45 \mathrm{~mm}$ sheath was introduced over a 0.035 inch hydrophilic soft guide wire from the right patent iliac site for angiographic visualization of the native aorta, the graft's main body, and the contralateral limb (left), which presented occlusion (Fig. 1, arrow). A hydrophilic soft guide wire (Terumo) was advanced centrally from the contralateral side (Fig. 1, right arrow) in a retrograde fashion. Since both central (graft main body-to-native-aorta) and left peripheral (graft limbto-common iliac artery) anastomoses were constructed in an end-to-side fashion, the manipulation and support provided by a guiding catheter (HeadHunter $\mathrm{H} 1$; Cordis/ Johnson \& Johnson, Miami, FL) assisted successfully the passage of the guide wire through these anastomoses and the thrombosed graft limb.

Accordingly, after systemic anticoagulation of the patient with $5000 \mathrm{IU}$ bolus heparin, a $9 \mathrm{~mm} \times 4 \mathrm{~cm}$ balloon was repeatedly inflated in the thrombosed limb, supported by the simultaneous inflation of a second balloon contralaterally. Failure to restore ade- quate perfusion was followed by deployment of a self-expanding vascular stent, $10 \mathrm{~mm} \times 10 \mathrm{~cm}$ (Bard E Luminexx), in the graft limb, with its proximal end protruding cephalad into the lumen of the main body (Fig. 2, arrow) above the level of the graft bifurcation, supported by the simultaneous inflation of the contralateral, occlusive balloon. Following deployment, the apposition of the stent was performed with simultaneous inflation of both balloons in a "kissing" technique (Fig. 2). The intraoperative angiography identified a stenosis of the peripheral anastomosis as the cause of thrombosis. Therefore, the stent extended below that level, involving the peripheral anastomosis (Fig. 3). No residual thrombus or residual stenosis was detected angiographically on completion angiography, and the internal iliac artery remained patent (Fig. 3), perfused through the struts of the stent. Optimal restoration of adequate flow to the periphery (Fig. 3) was ensured immediately postoperatively, with palpable pulses over the left dorsalis pedis artery and anklebrachial index of 1.1. The patient was discharged with dual antiplatelet therapy for 1 mo, followed by lifelong monotherapy with acetylsalicylic acid $(100 \mathrm{mg})$. He remains asymptomatic after $1-y r$ of follow-up with good palpable pulses.

\section{Discussion}

The technique of primary stenting for complete graft-limb occlusion after aortoiliac reconstructive sur- 

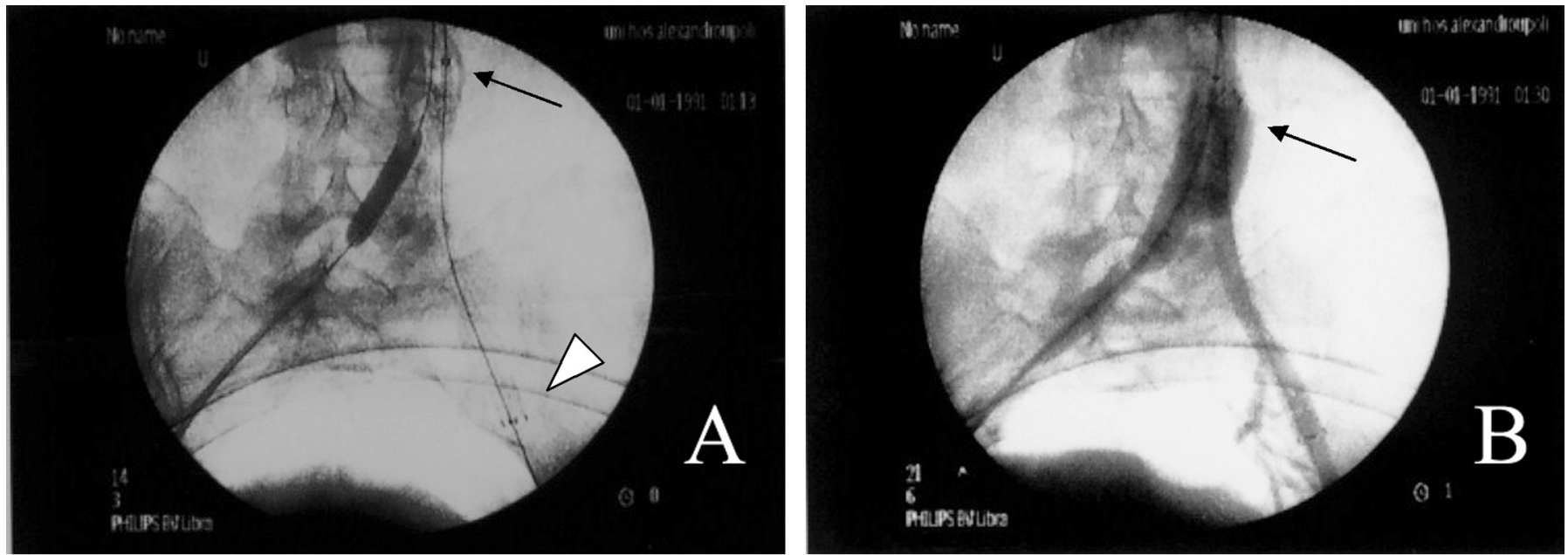

Figure 2. (A) Deployment of a self-expanding stent within the occluded graft limb. The central end of the stent is placed within the lumen of the main body, above the bifurcation level (arrow), whereas its peripheral end (white arrowhead) is located in the external iliac artery, thus involving the peripheral anastomosis. (B) Successful recanalization is accomplished in the graft limb.
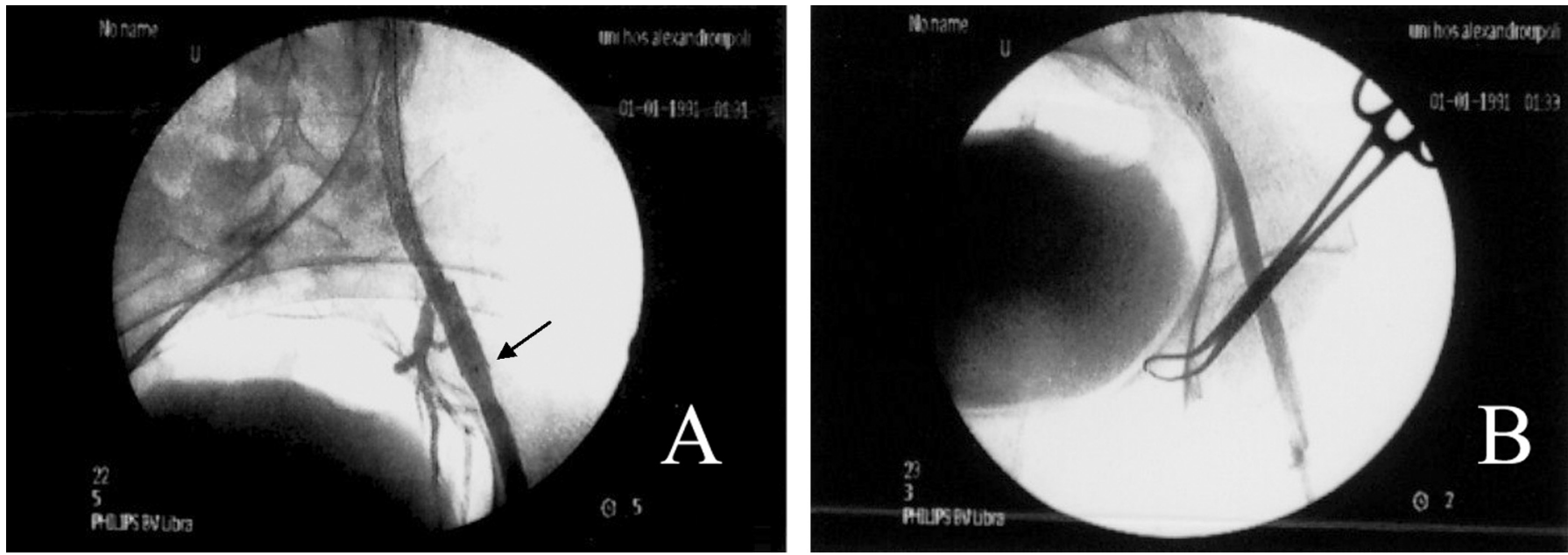

Figure 3. (A) The peripheral end of the stent (arrow), located in the external iliac artery. The orifice of the internal iliac artery is directly perfused through the stent struts. (B) Unimpaired flow to the level of the common femoral artery.

gery was recently described by Kondo et al. [3], who managed to recanalize a chronically thrombosed graft-limb of a bifurcated graft used for surgical repair of an abdominal aortic aneurysm (AAA). Although predilation is not recommended, in order to avoid plaque shift or distal embolism, we performed predilation in the occluded limb by using the simultaneous contralateral support of a dilated balloon, as recently described, to avoid the aforementioned risks and also to better support either the predilation or the stenting of the limb [4].
Although we initially attempted sole management with balloon inflation of the graft-limb, this was accompanied by significant recoil, making necessary the use of stenting to restore patency. Admittedly, primary stenting constitutes the best initial approach, since it could theoretically capture any thrombotic material, especially in cases of early thrombosis, where the thrombus is fresh and, thus, less organized. Apart from recanalization, primary stenting prevents recoil of the graft and distends the anastomotic sites where the cause is often detected, especially in early cases, 
like ours, preventing reocclusion after graft salvage. We chose a self-expandable stent instead of a balloonexpandable stent, since our technical target was to dilate a compliant graft-limb orifice, which differs from the calcified, noncompliant, tight-stenosed lesions of the native iliac ostia, requiring balloon-expandable stents.

The safety of stent grafts in prevention of distal embolization sounds appealing. However, in everyday practice, the use of self-expandable stents is preferable, since the feasibility for early intraoperative angiographic detection of peripheral embolization along with immediate intervention (thrombolysis or thrombectomy) render its use acceptable and safe. Large clinical series have described efficient recanalization of aortoiliac occlusion with extensive self- or balloonexpandable stenting, with quite low incidence of peripheral embolization, preserving stent grafts only for cases of arterial perforation [5-7]. Moreover, stent grafts also present the disadvantage of increased costs and collateral covering.

An additional difficulty in our case was the challenging passage of the guide wire from two end-toside anastomoses (centrally and peripherally), since the graft had been placed for aortoiliac disease, rather than AAA, where at least the central anastomosis is constructed in an end-to-end fashion, thus facilitating the guide wire passage; we were able to easily overcome the aforementioned difficulty with the support of a guiding catheter (e.g., HeadHunter). Furthermore, since the catheter is advanced only through thrombotic material occupying the entire graft limb, rather than calcified, hard stenotic lesions, the technical difficulty is rather mediated, making the passage technique feasible without being time-consuming. For these reasons, we recommend an initial attempt through the femoral access in order to recanalize a thrombotic graft limb in- stead of the brachial access, which may be troubling for some vascular surgeons.

Since major complications related to either catheter-directed thrombolytic therapy or an open-surgery approach are avoided, the endovascular approach seems attractive and feasible not only in early- or late-postoperative occlusions, but also in cases of acute ischemia, where the prolonged thrombolysis cannot be tolerated $[8,9]$. Generally, in aortoiliac failed grafts, angioplasty and/or stent placement follows catheter-directed thrombolysis, aspiration thrombectomy, or other clot removal methods, preceding stenting that aims mainly at correcting an anastomotic failure [10]. Our case represents a more straightforward single-step endovascular approach to thrombosed graft recanalization.

The long follow-up results remain an issue for such endovascular interventions, the efficacy of which should be further delineated. Notably, one year after the recanalization procedure, our patient has palpable femoral pulses with ankle-brachial index of 1.1 and excellent walking capacity.

To conclude, our example advocates the use of primary stenting as an effective therapeutic option for recanalization of a thrombosed aortic graft limb. This technique should be included in the armamentarium of every interventional specialist. Further comparative studies are needed in order to define whether this alternative can be considered as a first-line treatment for such cases.

\section{Conflict of Interest}

The authors have no conflict of interest relevant to this publication.

Comment on this Article or Ask a Question

\section{References}

1. Qu Z, Chaikof EL. Prosthetic grafts. In: Cronenwett JL, Johnston W, eds. Rutherford's Vascular Surgery, 7th ed. Philadelphia: Saunders Elsevier; 2010:1335-1349.

2. Pappy R, Hanna EB, Hennebry TA. Bifurcated aortic endograft limb occlusion managed with a novel method of isolated pharmacomechanical thrombectomy. Catheter Cardiovasc Interv. 2010;76:895-900. 10.1002/ccd.22645

3. Kondo Y, Dardik A, Muto A, Nishibe M, Nishibe T. Primary stent placement for late complete occlusion after aortoiliac reconstructive sur- gery: report of a case. Surg Today. 2009;39: 418-420. 10.1007/s00595-008-3855-y

4. Bechara CF, Barshes NR, Lin PH, Kougias P. Recanalization of flush iliac occlusions with the assistance of a contralateral iliac occlusive balloon. J Vasc Surg. 2012;55:872-874. 10.1016/j.jvs.2011.10.029

5. Kim TH, Ko YG, Kim U, Kim JS, Choi D, Hong $\mathrm{MK}$, et al. Outcomes of endovascular treatment of chronic total occlusion of the infrarenal aorta. J Vasc Surg. 2011;53:1542-1549. 10.1016/j.jvs.2011.02.015
6. Bin Jabr A, Sonesson B, Lindblad B, Dias N, Resch T, Malina M. Chimney grafts preserve visceral flow and allow safe stenting of juxtarenal aortic occlusion. J Vasc Surg. 2013; 57:399-405. 10.1016/j.jvs.2012.08.108

7. Moise MA, Alvarez-Tostado JA, Clair DG, Greenberg RK, Lyden SP, Srivastava SD, et al. Endovascular management of chronic infrarenal aortic occlusion. J Endovasc Ther. 2009; 16:84-92. 10.1583/08-2526.1

8. Anzuini A, Chiesa R, Airoldi F, Gimelli G, Civilini E, Dordoni L, et al. Regional throm- 
bolytic therapy and stent implantation in an acutely occluded aortobifemoral bypass graft. J Endovasc Ther. 2003;10:86-89. 10. 1583/1545-1550(2003)010<0086:RTTASI $>2$. $0 . \mathrm{CO} ; 2$

9. Rabellino M, García-Nielsen L, Gonzalez G, Baldi S, Zander T, Zerolo I, et al. Primary stent in occluded femoropopliteal bypass graft in a patient with contraindications for throm- bolytic therapy: case report. Cardiovasc Intervent Radiol. 2010;33:441-443. 10.1007/ s00270-009-9656-X

10. Eisenberg JA, Calligaro KD, Kolakowski S, Doerr KJ, Bennett S, Murtha K, et al. Is balloon angioplasty of peri-anastomotic stenoses of failing peripheral arterial bypasses worthwhile? Vasc Endovascular Surg. 2009;43: 346-351. 10.1177/1538574409336479
Cite this article as: Georgakarakos E, Kapoulas K. Percutaneous Endovascular Recanalization of a Thrombosed Aortic Graft Limb with Stent Placement. Aorta 2014;2(3):116-120. DOI: http://dx.doi. org/10.12945/j.aorta.2014.14-010 